\title{
Validación de la escala de autoeficacia general en Chile
}

\author{
PATRICIA CID H. ${ }^{\text {a }}$, ALDA ORELLANA Y. ${ }^{\text {la }}$, OMAR BARRIGA $^{2 b}$
}

\section{General self-efficacy scale validation in Chile}

Background: Self efficacy refers to an individual's belief in his or her capability to produce given achievements and the individual's perception of his or her ability to perform an action. Aim: To evaluate the psychometric properties of the General Self-efficacy Scale in Chilean population. Material and Methods: The study was carried out in 360 subjects, both sexes, 15-65 years of age, from Concepcion, Chile, who answered Self-efficacy, Self-esteem and Health Status Perception instruments. Reliability was verified by Cronbach's alpha coefficient and validity by expert revision, univariate statistics, correlations among items, item-scale correlations, and correlations with Self-esteem and Perception of Health Status constructs. Results: The structure of the scale is uni-dimensional, homogenous and positively related with the constructs examined. Conclusions: The General Self-efficacy Scale is a reliable and valid measure of the perception of self-efficacy in the Chilean population.

(Rev Med Chile 2010; 138: 551-557).

Key words: Reproducibility of results; Self concept: Self efficacy.

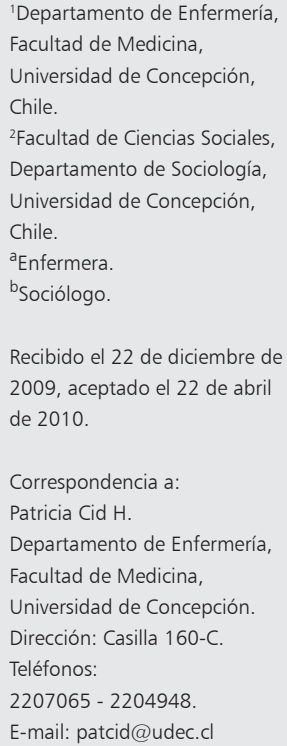

$\mathrm{B}$ andura inició el estudio de la autoeficacia en la década de 1970-79, motivado por la importancia que tiene el pensamiento autorreferente en la conducta de las personas. El conocimiento que tiene cada persona de su capacidad, la estimula o inhibe a realizar una determinada acción. Este autor definió la percepción de autoeficacia o autoeficacia percibida como los juicios de cada persona sobre sus capacidades, en base a los cuales organizará y ejecutará sus actos de modo que le permitan alcanzar el rendimiento deseado $^{1-2}$.

La autoeficacia percibida, en el ámbito de la salud, cobra importancia en la prevención de conductas riesgosas y en la promoción de conductas que van en beneficio de las personas. Se ha observado que los cambios de conducta se realizan en forma activa cuando los profesionales de la salud motivan en las personas su capacidad de iniciar$\operatorname{los}^{3}$. La autoeficacia ha sido estudiada desde dos perspectivas: la autoeficacia específica, definida como la creencia sobre el nivel de competencia en situaciones particulares, y la general, que se refiere a la sensación de competencia total de la persona que la habilita para enfrentar nuevas tareas y hacer frente a una gran variedad de situaciones difíciles ${ }^{4}$.

Un instrumento que mide la autoeficacia general es el desarrollado en 1979 por Schwarzer y Jerusalem en Alemania, el cual mide la percepción que tiene la persona respecto de sus capacidades para manejar en su vida diaria diferentes situaciones estresantes 5 . Más tarde, en 1981, reducen esta versión alemana de 20 a 10 reactivos, siendo traducida a 28 idiomas $^{6,7}$.

La versión al español se tradujo por expertos en 1993 desde la escala original, con adaptaciones culturales necesarias para medir de manera contextualizada el constructo autoeficacia percibida en población costarricense, española y peruana ${ }^{6,8-11}$.

Los estudios de las características psicométricas de la Escala de Autoeficacia General (EAG) en español, han demostrado empíricamente su confiabilidad y validez convergente y discriminante. La unidimensionalidad y homogeneidad de las versiones en español, chino y alemán se demostró a través del análisis de la correlación ítem-ítem 
total, factor carga y componentes principales ${ }^{6,8-11}$.

El constructo autoeficacia percibida se ha correlacionado positivamente con optimismo, autoestima, autorregulación, calidad de vida, afectos positivos, competencia percibida, personalidad resistente, afrontamiento centrado en la tarea y satisfacción en el trabajo/colegio ${ }^{6-7,9}$. Por otro lado, negativamente con depresión y ansiedad ${ }^{6,7}$.

Considerando la importancia de este constructo en los cambios de conducta en salud y los positivos resultados psicométricos de la EAG obtenidos en otros países, esta investigación tiene como objetivo evaluar características psicométricas de la Escala Autoeficacia General en población chilena, para contar con un instrumento preciso de medida de autoeficacia percibida para el país.

\section{Material y Método}

Estudio realizado con datos obtenidos desde una muestra de 360 personas de ambos sexos, en edades comprendidas entre 15 y 65 años de la Comuna de Concepción (Chile) ${ }^{12}$. Se aplicó en entrevista personalizada; las escalas de Autoeficacia General, de Autoestima y de Estado de Salud Percibido, previo consentimiento informado.

Para llevar a cabo la evaluación psicométrica de la EAG en español, en esta investigación se analizó la confiabilidad y validez del instrumento. La confiabilidad se estudió a través de la consistencia interna utilizando el coeficiente de confiabilidad alfa de Cronbach que mide la correlación promedio entre reactivos y el número de reactivos de un determinado instrumento o cómo el constructo está representado en cada reactivo ${ }^{13-15}$. Este coeficiente oscila entre $-1,0$ y $+1,0$, y se consideró que los reactivos medían en forma óptima el constructo cuando sus valores fluctuaban entre 0,7 y $0,9^{15-16}$.

La validez del instrumento se analizó a través de validez de constructo y de criterio siguiendo los planteamientos de Hernández, Fernández y Baptista (2003), quienes definen la validez de un instrumento como "el grado en que un instrumento realmente mide la variable que pretende medir" (p. 346) ${ }^{17}$. Estos autores adaptan las recomendaciones dadas en la década 1950-59 por el Comité de Tests Psicológicos de la American Psychological Association con respecto al proceso de validación de los instrumentos que miden constructos psicológicos antes de su publicación ${ }^{18}$.

Inicialmente la EAG se llevó a revisión por expertos del área de Psicología, Sociología y Enfermería para asegurar la comprensión de los reactivos, posteriormente se realizó prueba piloto del instrumento en la cual 40 personas mostraron una buena comprensión de la escala.

La validez del constructo autoeficacia percibida, se analizó por medio de los estadísticos de los elementos, correlación entre los elementos y, entre elemento y escala total; y correlación con los constructos autoestima y estado de salud percibido (validez de criterio concurrente).

\section{Instrumentos}

La Escala de Autoeficacia General versión en español de Bäßler, Schwarzer y Jerusalem (1993) se conforma de 10 reactivos con un puntaje mínimo de 10 puntos y un máximo de 40 puntos $^{8}$. Las respuestas son tipo Likert donde la persona responde a cada reactivo de acuerdo a lo que ella percibe de su capacidad en el momento: Incorrecto (1 punto); apenas cierto ( 2 puntos); más bien cierto ( 3 puntos) o cierto (4 puntos $)^{6,19}$. En esta escala a mayor puntaje mayor autoeficacia general percibida.

La Escala de Autoestima de Morris Rosenberg (1965) mide la valoración que hace la persona de si misma, diseñada con 10 ítems y un formato de respuesta tipo likert de 1 a 4 puntos, resultando una escala de 10 a 40 puntos. La mayor autoestima de la persona está representada por el puntaje más alto ${ }^{20,21}$.

La Escala de Estado de Salud Percibido o Percepción de Bienestar de Reker y Wong (1984) mide la autovaloración que la persona hace de su estado de salud. Escala tipo Likert de 16 ítems, donde las respuestas van de "muy de acuerdo" a "muy en desacuerdo", con valores de 7 a 1 punto respectivamente. El puntaje total de la escala oscila de 112 a 16 puntos, donde 112 refleja un mejor estado de salud percibido ${ }^{22}$.

\section{Resultados}

\section{Análisis descriptivo de la EAG}

En el análisis descriptivo de los puntajes empíricos en este estudio de autoeficacia percibida, se observó que las respuestas de las personas calificaron con un mínimo de 16 y un máximo de 40 puntos y un rango de 24 puntos entre ambos. De acuerdo al valor de la media y de la mediana, las personas en general, se perciben con una 
Validación de la escala de autoeficacia general en Chile - P. Cid H. et al

buena capacidad de ejecutar una acción, apoyada también por la variabilidad de la suma del total del puntaje que osciló entre 29,34 y 39,02 puntos. Los valores de tendencia central representan una distribución de los datos que no responden a una curva normal, con una desviación de la curva a la izquierda y con observaciones agrupada hacia la derecha. De acuerdo al coeficiente de curtosis la curva es de tipo mesocúrtica (Tabla 1).

\section{Confiabilidad de la EAG}

El coeficiente alfa de Cronbach de la EAG $(0,84)$ indica que $84 \%$ de la variabilidad de las puntuaciones obtenidas representa diferencias verdaderas entre las personas y $16 \%$ refleja fluctuaciones al azar. Este resultado nos permite aseverar que los reactivos o elementos son homogéneos y que la escala mide de forma consistente la característica para la cual fue elaborada (Tabla 2).
Tabla 1. Estadísticos descriptivos de la EAG

\begin{tabular}{|lc|}
\hline $\mathrm{n}$ & 360 \\
\hline Media & 34,18 \\
\hline Mediana & 35 \\
\hline Moda & 40 \\
\hline Desianza & 23,51 \\
\hline Asimetría & 4,84 \\
\hline Curtosis &,- 78 \\
\hline Mínimo &, 13 \\
\hline Máximo & 16 \\
\hline Rango & 40 \\
\hline
\end{tabular}

Tabla 2. Estadísticos EAG total-elemento

\begin{tabular}{|c|c|c|c|c|}
\hline & $\begin{array}{l}\text { Media de la } \\
\text { escala si se } \\
\text { elimina el } \\
\text { elemento }\end{array}$ & $\begin{array}{l}\text { Varianza de } \\
\text { la escala si } \\
\text { se elimina } \\
\text { el elemento }\end{array}$ & $\begin{array}{l}\text { Correlación } \\
\text { elemen- } \\
\text { to- total } \\
\text { corregida }\end{array}$ & $\begin{array}{l}\text { Alfa de } \\
\text { Cronbach si } \\
\text { se elimina } \\
\text { el elemento }\end{array}$ \\
\hline $\begin{array}{l}\text { 1. Puedo encontrar la manera de obtener lo } \\
\text { que quiero aunque alguien se me oponga }\end{array}$ & 30,85 & 19,45 & 0,44 & 0,84 \\
\hline $\begin{array}{l}\text { 2. Puedo resolver problemas difíciles si me } \\
\text { esfuerzo lo suficiente }\end{array}$ & 30,43 & 21,17 & 0,46 & 0,83 \\
\hline $\begin{array}{l}\text { 3. Me es fácil persistir en lo que me he pro- } \\
\text { puesto hasta llegar a alcanzar mis metas }\end{array}$ & 30,84 & 19,59 & 0,49 & 0,83 \\
\hline $\begin{array}{l}\text { 4. Tengo confianza en que podría manejar } \\
\text { eficazmente acontecimientos inesperados }\end{array}$ & 30,83 & 18,42 & 0,61 & 0,82 \\
\hline $\begin{array}{l}\text { 5. Gracias a mis cualidades y recursos puedo } \\
\text { superar situaciones imprevistas }\end{array}$ & 30,75 & 19,34 & 0,54 & 0,83 \\
\hline $\begin{array}{l}\text { 6. Cuando me encuentro en dificultades pue- } \\
\text { do permanecer tranquilo/a porque cuento } \\
\text { con las habilidades necesarias para manejar } \\
\text { situaciones difíciles }\end{array}$ & 31,03 & 18,04 & 0,58 & 0,82 \\
\hline $\begin{array}{l}\text { 7. Venga lo que venga, por lo general soy } \\
\text { capaz de manejarlo }\end{array}$ & 30,88 & 18,33 & 0,69 & 0,81 \\
\hline $\begin{array}{l}\text { 8. Puedo resolver la mayoría de los problemas } \\
\text { si me esfuerzo lo necesario }\end{array}$ & 30,46 & 20,45 & 0,58 & 0,82 \\
\hline $\begin{array}{l}\text { 9. Si me encuentro en una situación difícil, } \\
\text { generalmente se me ocurre qué debo hacer }\end{array}$ & 30,73 & 19,29 & 0,57 & 0,82 \\
\hline $\begin{array}{l}\text { 10. Al tener que hacer frente a un problema, } \\
\text { generalmente se me ocurren varias alterna- } \\
\text { tivas de cómo resolverlo }\end{array}$ & 30,78 & 19,65 & 0,49 & 0,83 \\
\hline
\end{tabular}


Tabla 3. Estadísticos de resumen de los elementos de la EAG

\begin{tabular}{|lccccccc|}
\hline & Media & Mínimo & Máximo & Rango & $\begin{array}{c}\text { Máximo/ } \\
\text { mínimo }\end{array}$ & Varianza & Elementos \\
\hline $\begin{array}{l}\text { Medias de los } \\
\text { elementos }\end{array}$ & 3,418 & 3,144 & 3,750 & 0,606 & 1,193 & 0,035 & 10 \\
$\begin{array}{l}\text { Varianza de los } \\
\text { elementos }\end{array}$ & 0,565 & 0,244 & 0,859 & 0,616 & 3,526 & 0,035 & 10 \\
$\begin{array}{l}\text { Covarianzas } \\
\text { Inter-elementos }\end{array}$ & 0,199 & 0,084 & 0,454 & 0,370 & 5,428 & 0,006 & 10 \\
$\begin{array}{l}\text { Correlaciones } \\
\text { Inter-elementos }\end{array}$ & 0,361 & 0,199 & 0,633 & 0,435 & 3,187 & 0,008 & 10 \\
\hline
\end{tabular}

Tabla 4. Matriz de correlaciones inter-elementos de la EAG

\begin{tabular}{|c|c|c|c|c|c|c|c|c|c|c|}
\hline Elementos & 1 & 2 & 3 & 4 & 5 & 6 & 7 & 8 & 9 & 10 \\
\hline \multicolumn{11}{|l|}{1} \\
\hline 2 & 0,199 & & & & & & & & & \\
\hline 3 & 0,390 & 0,351 & & & & & & & & \\
\hline 4 & 0,351 & 0,279 & 0,354 & & & & & & & \\
\hline 5 & 0,291 & 0,316 & 0,340 & 0,487 & & & & & & \\
\hline 6 & 0,241 & 0,304 & 0,242 & 0,460 & 0,427 & & & & & \\
\hline 7 & 0,310 & 0,382 & 0,382 & 0,503 & 0,429 & 0,633 & & & & \\
\hline 8 & 0,328 & 0,417 & 0,314 & 0,472 & 0,282 & 0,405 & 0,484 & & & \\
\hline 9 & 0,298 & 0,310 & 0,309 & 0,395 & 0,290 & 0,391 & 0,476 & 0,429 & & \\
\hline 10 & 0,264 & 0,255 & 0,268 & 0,301 & 0,291 & 0,337 & 0,389 & 0,350 & 0,509 & \\
\hline
\end{tabular}

\section{Validez del constructo autoeficacia percibida}

En la Tabla 3 se presentan los estadísticos de resumen de los diez elementos de la EAG, donde 3,41 fue el puntaje promedio, oscilando entre 3,14 y 3,75 , evidenciando una estructura de respuesta de puntaje alto ("más bien cierto" y "cierto"). La correlación promedio entre-elementos $(0,36)$ demuestra que existe una relación positiva, pero de acuerdo al rango $(0,43)$ de esta relación hace dudar en primera instancia de la homogeneidad entre los elementos.

Esta duda se descarta al analizar la matriz de correlaciones (Tabla 4), donde se aprecia que sólo existen dos pares de elementos con valores extremos $(0,19-0,63)$, en cambio, las coeficientes de los otros pares de elementos oscilan en valores cercanos entre sí $(0,24-0,50)$. Los elementos que más se correlacionan son el 6 "cuando me encuen- tro en dificultades puedo permanecer tranquilo/a porque cuento las habilidades necesarias para manejar situaciones difíciles" con el 7 "venga lo que venga, por lo general soy capaz de manejarlo". Los elementos que menos se correlacionan son el 1 y el 2, "puedo encontrar la manera de obtener lo que quiero aunque alguien se me oponga" y "puedo resolver problemas difíciles si me esfuerzo lo suficiente", respectivamente.

En la Tabla 2 se aprecia que cada uno de los 10 elementos se correlacionan positivamente con la EAG total, el elemento 1 es el que menos contribuye en la EAG $(0,444)$, con respecto al elemento $7(0,693)$.

A la vez se observa que el coeficiente de confiabilidad disminuye al extraer cualquiera de los 10 reactivos o elementos, destacando que la eliminación del reactivo 7 es el que más afecta la 


\section{Tabla 5. Correlaciones entre percepción de autoeficacia con autoestima, y con estado de salud percibido}

\begin{tabular}{|lcc|}
\hline Escala & $\begin{array}{c}\text { Correlación con } \\
\text { autoeficacia }\end{array}$ & $\mathbf{n}$ \\
\hline Autoestima &, $305^{* *}$ & 360 \\
$\begin{array}{l}\text { Estado de salud } \\
\text { percibido }\end{array}$ &, $159^{* *}$ & 360 \\
\hline
\end{tabular}

**La correlación es significativa al nivel 0,01 (bilateral).

confiabilidad de la EAG $(0,844$ a 0,815), disminuyendo el coeficiente de confiabilidad en casi 0,03 puntos, por lo tanto ninguno de los elementos se pueden eliminar para fortalecer la escala (Tabla 2).

\section{Validez de criterio concurrente:}

La validez de criterio concurrente entre EAG con las escalas de Autoestima y Estado de Salud Percibido:

La correlación observada entre la EAG y la escala de Autoestima tiene una magnitud media positiva de $0,305(p \leq 0,01)$. Menor es el valor de correlación $(\mathrm{r}=0,159)$ entre la EAG y la escala de Estado de Salud Percibido, al mismo nivel de significancia (Tabla 5).

\section{Discusión}

Los hallazgos del estudio demuestran las adecuadas características psicométricas de la EAG en población chilena de ambos sexos, entre 15 y 65 años. Con respecto a la confiabilidad de la EAG, se demostró su consistencia interna u homogeneidad al obtener un alto coeficiente alfa de Cronbach, similar a los resultados obtenidos en los estudios que utilizaron este mismo instrumento en población costarricense, española y peruana ${ }^{9-11}$. La confiabilidad de la EAG en este estudio es muy cercana a la encontrada en el idioma original ${ }^{6}$.

Los estadísticos para validar el constructo autoeficacia percibida reflejan relaciones que apuntan en la misma dirección entre los pares de elementos, y muestran que cada uno de los 10 elementos tiene una contribución particular en el puntaje total, confirmándose la característica unidimensional de la escala. Característica ya observada en investigaciones anteriores que incluyeron la versión de la escala en español ${ }^{6,11}$.
Específicamente los reactivos 1 y 7 , "puedo encontrar la manera de obtener lo que quiero aunque alguien se me oponga" y "venga lo que venga, por lo general soy capaz de manejarlo" presentaron comportamientos semejantes en la validación de la escala en población española9.

Las correlaciones positivas de Autoeficacia Percibida con Autoestima y Estado de Salud Percibido apoyan la validez del constructo en este estudio, esta relación se refleja en que a medida que aumenta la autoeficacia general de la población estudiada también aumenta su autoestima y su estado de salud percibido o percepción de bienestar. Relaciones en la misma dirección se reflejan en un estudio sobre la conducta de fumar en adolescentes, donde los que no fumaban percibían mayor autoestima y autoeficacia que los adolescentes fumadores y en un estudio donde los investigadores demostraron que la autoeficacia percibida puede aumentarse a través de intervenciones que logran muy buenos resultados en la percepción de bienestar de las personas con artritis ${ }^{23-27}$.

La correlación obtenida entre los constructos de autoeficacia y autoestima demuestran que ambos son evaluaciones subjetivas personales que se relacionan, pues la autoeficacia es una medida de capacidad personal y la autoestima de valía personal influida por los cánones sociales y los criterios personales. Bandura expresa al respecto "en la mayoría de las actividades que emprende el individuo cultiva su autoeficacia en aquellas que le dan sensación de autovalía” (p. 435) ${ }^{1}$.

Por otro lado, la percepción de bienestar está relacionada con las emociones y vitalidad de las personas, y autoeficacia percibida influye en la respuesta emocional y en la actitud al cambio de conducta en salud, dando apoyo a la correlación obtenida entre ambos constructos ${ }^{1,28}$.

Respecto a la autoeficacia percibida de la población estudiada, esta es similar a la informada en población costarricense, y más alta que en otras culturas ${ }^{7}$. Este hallazgo es muy importante en el sentido que en general las personas de este estudio se creen capaces de involucrarse en un cambio, y específicamente esto facilitará motivar a las personas a que se involucren en una conducta particular, como por ejemplo: motivar para cambiar la conducta fumadora o prevenirla al resistir la presión de los pares para su inicio, el uso del condón como conducta preventiva contra la infección del VIH, el auto-examen de mamas para la 
detección precoz del cáncer de mama, para asumir una enfermedad como la artritis y los cambios que ella conlleva, como también fortalecer la capacidad de los cuidadores de familiares con demencia ${ }^{29-34}$.

Se puede concluir que la Escala de Autoeficacia General es fiable y válida para medir el constructo autoeficacia percibida en población chilena y similar a los resultados obtenidos en estudios en poblaciones de habla hispana. Por tanto, se afirma que esta escala de medición de autoeficacia tiene correspondencia con la realidad del fenómeno que se estudia ${ }^{35}$.

\section{Referencias}

1. Bandura A. Autoeficacia. En: Pensamiento y acción: fundamentos sociales. Barcelona: Martínez Roca SA, 1987; 415-78.

2. Bandura A. Self-efficacy: Toward a Unifying theory of behavioral change. Psychological Rev 1977; 84 (2): 191215.

3. Villamarín F, Sanz A. Autoeficacia y salud: investigación básica y aplicaciones. En: Salanova M, Grau R, Martínez I, Cifre E, Llorens S, García-Renedo M. Nuevos horizontes en la investigación sobre autoeficacia. Castelló de la Plana: Publicacions de la Universitat Jaume I, 2004: 119-32.

4. Grau R, Salanova M, Peiró JM. Efectos moduladores de la autoeficacia en el estrés laboral. Apuntes de Psicología 2000; 18 (1): 57-75.

5. Schwarzer R, Jerusalem W. Generalized self-efficacy scale. In: J. Weinman, J. Wright and M. Johnston. Measures in health psychology: A user's portfolio. Causal and control beliefs. Windsor, England. WFER-WELSON 1995; 35-37.

6. Schwarzer R, Bäßler J, Kwiatek P, Schröder K, Zhang JX. The assessment of optimistic self-beliefs: Comparison of the german, spanish, and chinese versions of the general self-efficacy scale. Applied Psychology 1997; 46 (1): 6988.

7. Luszczynska A, Gutiérrez-Doña B, Schwarzer R. General self-efficacy in various domains of human functioning: Evidence from five countries. International Journal of Psychology 2005; 40 (2): 80-9.

8. Baessler J, Schwarzer R. Evaluación de la autoeficacia: Adaptación española de la escala de autoeficacia general. Ansiedad y estrés 1996; 2 (1): 1-8.

9. Sanjuán P, Pérez A, Bermúdez J. Escala de autoeficacia general: datos psicométricos de la adaptación para población española. Psicothema 2000; 12 (suppl 2): 509-13.

10. Klassen R. Optimism and realism: A review of self- efficacy from a cross-cultural perspective. International Journal of Pschycology 2004; 39: 205-30.

11. Scholz U, Gutiérrez-Doña B, Sud S, Schwarzer R. Is general self-efficacy a universal construct? Psychometric findings from 25 countries. European Journal of Psychological Assessment 2002; 18 (3): 242-51.

12. Cid P, Merino JM, Stiepovich J. Factores biológicos y psicosociales predictores del estilo de vida promotor de salud. Rev Med Chile 2006; 134 (12): 1491-9.

13. Nunnally J, Bernstein I. La evaluación de la confiabilidad. En: Nunnally J, Bernstein I. Teoría psicométrica. $3^{a}$ edición. México: Mc Graw Hill, 1995: 277-326.

14. Oviedo H, Campo-Arias A. Aproximación al uso del coeficiente alfa de Cronbach. Revista Colombiana de Psiquiatría 2005; 34 (4): 572-80.

15. Campo-Arias A. Usos del Coeficiente de Alfa de C. Cronbach (carta al editor). Biomédica 2006; 26 (4): 5858.

16. Pérez C. Reducción de la dimensión: fiabilidad de escalas y escalamiento multidimensional. En: Pérez C. Métodos estadísticos avanzados con SPSS. Madrid: Thomson, 2005; 689-758.

17. Hernández R, Fernández C, Baptista P. Recolección de los datos. En: Hernández R, Fernández C, Baptista P. Metodología de la Investigación. $3^{\text {a }}$ edición. México: Mc Graw Hill 2003; 342-482.

18. Miljánovich M. Validez de constructos hipotéticos en psicología. Revista de Psicología 1997; 1 (1). Disponible en http://sisbib.unmsm.edu.pe/BvRevistas/psicologia/1997_n1/validez.htm. Accesado: 16 de mayo de 2008.

19. Schwarzer R. Spanish Adaptation of the General SelfEfficacy Scale. Disponible en http://userpage.fu-berlin. de/ health/spanscal.htm Accesado: 16 de mayo de 2008.

20. Rosenberg M. The self-esteem scala. En: Blascovich J y Tomaka J. Measures of self-esteem. En: Robinson, J. Shaver, P. Wrightman, L. Measures of Personality and Social Psychological Attitudes. Academic Press. United States of América 1991; 121-3.

21. Vásquez A, Jiménez R, Vásquez R. Escala de autoestima de Rosenberg: fiabilidad y validez en población clínica española. Apuntes de Psicología 2004; 22 (2): 247-56.

22. Reker GT, Wong P. Psychological and physical well-being in the elderly: the perceived well-being scale (PWB). Canadian Journal on Aging 1984; 3 (1): 23-32.

23. Chen G, Gully S, Eden D. General self-efficacy and selfesteem: toward theoretical and empirical distinction between correlated self-evaluations. Journal of organizational Behavior 2004; 25 (3): 375-95.

24. Luszczynska A, Scholz U, Schwarzer R. The General self-efficacy scale: Multicultural validation studies. The Journal of Psychology 2005; 139 (5): 439-57. 
25. Olivari C, Barra E. Influencia de la autoeficacia y la autoestima en la conducta de fumar en adolescentes. Terapia Psicológica 2005; 23 (2): 5-12.

26. Marks R, Allegrante J, Lorig K. A review and synthesis of research evidence for self-efficacy-enhancing interventions for reducing chronic disability: implications for health education practice (part I). Health Promotion Practice 2005; 6 (1): 37-43.

27. Marks R, Allegrante J, Lorig K. A review and synthesis of research evidence for self-efficacy-enhancing interventions for reducing chronic disability: implications for health education practice (part II). Health Promotion Practice 2005; 6 (2): 148-56.

28. Reker GT, Peacock P, Wong P. Meaning and purpose in life and well-being: a life-span perspective. J Gerontol 1987; 42 (1): 44-9.

29. Schwarzer R, Gutiérrez-Doña B. Health Psychology. En: Pawlik K, Rosenzweig MR. International Handbook of Psychology. London: Sage Publications, 2000: 452-65.

30. Sánchez-Zamorano L, Llerena A, Anaya-Ocampo R, Lazcano-Ponce E. Prevalencia del uno de drogas ilegales en función del consumo de tabaco en una muestra de estudiantes en México. Salud Pública de México 2007; 49 (suppl 2): 182-93.

31. Nieto-Andrade B, Izazola-Licea J. Uso del condón en hombres con parejas no estables en la Ciudad de México. Salud Pública de México 1999; 41 (2): 85-94.

32. Haas BK. Focus on health promotion: Self-efficacy in oncology nursing research and practice. Oncology Nursing Forum 2000; 27 (1): 89-97.

33. Vinaccia S, Contreras F, Restrepo L, Cadena J, Anaya J. Autoeficacia, desesperanza aprendida e incapacidad funcional en pacientes con diagnóstico de artritis reumatoide. Internacional Journal of Clinical and Health Psychology 2005; 5 (1): 129-42.

34. Steffen A, McKibbin C, Zeiss A, Gallagher-Thompson D, Bandura A. The revised scale for caregiving self-efficacy: reliability y validity studies. The Journals of Gerontology 2002; 57 (1): 74-86.

35. Sánchez R, Echeverry J. Validación de escalas de medición en salud. Revista de Salud Pública 2004; 6 (3): 302-18. 